\title{
POLAR SETS AND PALM MEASURES IN THE THEORY OF FLOWS( ${ }^{1}$ )
}

\author{
BY \\ DONALD GEMAN AND JOSEPH HOROWITZ
}

\begin{abstract}
Given a flow $\left(\theta_{t}\right), t$ real, over a probability space $\Omega$, we prove that certain measures on $\Omega$ (viewed as the state space of the flow) decompose uniquely into a Palm measure $Q$ which charges no "polar set" and a measure supported by a polar set. Considering the continuous and discrete parts of the additive functional corresponding to $Q$, we find that $Q$ further decomposes into a measure charging no "semipolar set" and a measure supported by one. As a consequence, Palm measures are exactly those which neglect sets which the flow neglects, and polar sets are exactly those neglected by every Palm measure. Finally, we characterize various properties, such as predictability and continuity, of an additive functional in terms of its Palm measure. These results further illuminate the role played by supermartingales in the theory of flows, as pointed by J. de Sam Lazaro and P. A. Meyer.
\end{abstract}

0 . Introduction. Let $\left(\Omega, \mathcal{F}_{t}^{0}, P, \theta_{t}\right), t \in \mathbf{R}$ (the real line), be a filtered dynamical system (all terminology will be explained below). In $\$ 1$ we prove that a finite measure $Q$ on $\mathcal{F}_{0-}^{0}$ which is "progressively absolutely continuous" decomposes uniquely into the sum of two measures $Q=P_{a}^{-}+\mu$, where $P_{a}^{-}$is the restriction to $\mathcal{F}_{0}^{0}$ of the Palm measure $P_{\alpha}$ of a predictable additive functional $\alpha$, and $\mu$ is supported by a "polar" set in $\mathcal{F}_{0-}^{0}$. Decomposing $\alpha$ into its continuous and discrete parts, say $\alpha_{c}$ and $\alpha_{d}$, we will see (in \$2) that $P_{a}^{-}$splits into a measure $P_{c}^{-}$which charges no "semipolar" set and a measure $P_{d}^{-}$which is carried by a semipolar, but charges no polar, set. Thus we have a decomposition

$$
Q=P_{c}^{-}+P_{d}^{-}+\mu
$$

analogous to that of a measure on the state space of a Markov process [1,

Received by the editors April 11, 1974.

AMS (MOS) subject classifications (1970). Primary 28A65, 60G10.

Key words and phrases. Flow, filtration, dynamical system, Palm measure, additive functional, polar set, semipolar set.

( ${ }^{1}$ ) This work was partially supported by National Science Foundation grant GP 34485 Al. 
p. 283]; this is not entirely surprising in view of the Markovian nature of the flow $\theta_{t}:\left(\Omega, \mathcal{F}_{t}^{0}\right) \rightarrow\left(\Omega, \mathcal{F}_{0}^{0}\right)$ (see [9]). The decomposition (1) requires the Doob-Meyer decomposition of supermartingales and is related to Föllmer's [3] correspondence between supermartingales and certain measures on $\mathbf{R}_{+} \times$ $\Omega$. As a corollary, we find that a finite measure $Q$ on $\bigvee_{t \in \mathrm{R}} \mathcal{F}_{t}^{0}$ is a Palm measure iff it charges no polar set. The section concludes with a characterization of polar sets, and several applications of these ideas, particularly to local times.

In $\$ 2$ we characterize various properties of a given additive functional $a$, such as well-measurability, predictability, and continuity, in terms of its Palm measure. Papangelou [14] has recently given some results on stationary point processes (which we construe as additive functionals which increase only by unit jumps) of the type we are considering. (Similar questions for Markov additive functionals have been treated by Revuz [16].) In the present article we will generalize several of Papangelou's results and obtain flow theory analogues of some of the Markovian ones. Some of our material will be recognized as a specialization of results in the "general theory of processes" [2], with more detail made possible by additional structure. In fact, our intention throughout is to solidify further the bridge built between the general theory and flow theory by J. de Sam Lazaro and P. A. Meyer [8], [9].

The remainder of this section is devoted to an explanation of the terminology and background material. Our notation is largely that of [1, Chapter 0], with this exception: if $(E, \mathcal{E})$ is a measurable space, we write (ambiguously) $f \in(\mathcal{E})$ to mean that $f$ is an $\mathcal{E}$-measurable function on $E$, the range being clear from context; $f \in(\tilde{E})_{+}$indicates the range is $\mathbf{R}_{+}=[0, \infty)$.

A flow $\theta=\left(\theta_{t}\right), t \in \mathbf{R}$, on a probability space $\left(\Omega, \mathcal{F}^{0}, P\right)$ is a oneparameter group (under composition) of bimeasurable, measure-preserving bijections $\theta_{t}: \Omega \rightarrow \Omega$ such that $\theta_{0}=$ identity and the mapping $(t, \omega) \rightarrow \theta_{t}(\omega)$ is $B \otimes \mathcal{F}^{0} / \mathcal{F}^{0}$-measurable. We further assume the existence of a filtration, i.e. an increasing family of $\sigma$-fields $\left\{\mathcal{F}_{t}^{0}\right\}, t \in \mathbf{R}$, on $\Omega$ whose generated $\sigma$ field $\bigvee_{t \in R} \mathcal{F}_{t}^{0}$ is $\mathcal{F}^{0}$, and which is compatible with the flow $\theta$ in that $\theta_{t}^{-1} \mathcal{F}_{s}^{0}=\mathscr{F}_{s+t}^{0}, s, t \in R$. As usual we write $\mathcal{F}_{t_{+}}^{0}=\bigcap_{s>t} \mathcal{F}_{s}^{0}, \mathcal{F}_{t-}^{0}=\bigvee_{s<t} \mathcal{F}_{s^{0}}^{0}$ Each $\mathcal{F}_{t}^{0}$ (and thus $\mathfrak{F}^{0}$ ) is assumed separable. The $P$-completion of $\mathscr{F}^{0}$ is denoted $\mathcal{F}_{\text {, and then }} \mathcal{F}_{t}$ is obtained by adjoining to $\mathcal{F}_{t}^{0}$ all sets in $\mathcal{F}$ of measure zero. The family $\left\{\mathcal{F}_{t}\right\}$ is then right-continuous [2] and compatible with $\theta$, but $\theta:(t, \omega) \rightarrow \theta_{t}(\omega)$ need not be $\mathbb{B} \otimes \mathcal{F} / \mathcal{F}$-measurable. The entity $\left(\Omega, \mathfrak{F}_{t}^{0}, P, \theta_{t}\right)$ is a filtered dynamical system. Concepts from the general theory of processes, such as predictability, are in reference to the family $\left\{\mathcal{F}_{t}\right\}$ unless otherwise indicated. 
An additive functional (AF) is a real-valued process $\alpha=\alpha(t, \omega)$ (or $\left.\alpha_{t}(\omega)\right), t \in \mathbf{R}, \omega \in \Omega$, such that (i) $\alpha(0)=0$; (ii) almost every path is rightcontinuous, nondecreasing; (iii) for each $s, t \in \mathbf{R}$ there is a set $N_{s t} \in \mathcal{F}$ of measure zero such that

$$
\alpha(t+s, \omega)=\alpha(t, \omega)+\alpha\left(s, \theta_{t} \omega\right)
$$

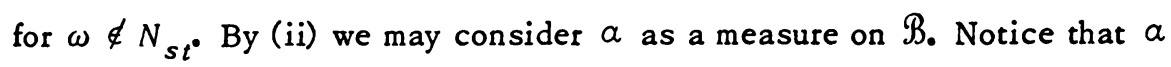
need not be adapted; sometimes we will use the phrase (due to Getoor and Sharpe) "raw additive functional" (RAF) to emphasize this point. We call a adapted if $\alpha(t) \in\left(\mathcal{F}_{t}\right)$ for $t \geq 0$, and this implies $\alpha(t) \in \mathcal{F}_{0}$ if $t \leq 0$. As shown in [9], there exists an AF $\bar{\alpha}$ indistinguishable from $\alpha$ (i.e. such that $\bar{\alpha}(t, \omega)=\alpha(t, \omega)$ for all $t \in \mathbf{R}$ a.s.) which is perfect in that the set $N_{s t}$ in (iii) may be chosen independently of $s, t, \mathcal{F}^{0}$-measurable, and such that (iv) $\bar{\alpha}\left( \pm_{\infty}, \omega\right)= \pm_{\infty}$ or $\bar{\alpha}(t, \omega) \equiv 0$ for every $\omega \in \Omega$.

Given an AF $a$, its Palm measure is

$$
P_{a}(A)=E \int_{0}^{1} I_{A} \circ \theta_{t} d \alpha_{t} \quad\left(=E \int_{0}^{\infty} e^{-t} I_{A} \circ \theta_{t} d \alpha_{t}\right), \quad A \in \mathcal{F}^{0} \text {, }
$$

where $I_{A}$ is the indicator of $A$. Palm measures arise naturally in the study of "flows under a function" [7], local times [4], "time-changes" of flows [17], point processes ("Palm-Khinhin formulae" etc.), and level crossings ("horizontal-window" probabilities). They are exactly the measures which neglect sets in $\Omega$ which the flow neglects (Theorem (10)).

Finally, we will need these facts (see [5], [9]). $P_{\alpha}$ is always $\sigma$-finite and finite iff $E \alpha(1)<\infty$, in which case $\alpha$ is called integrable and $E \alpha(t)=$ $t E \alpha(1)$. Two AF's $\alpha, \beta$ are indistinguishable iff their $\mathrm{Palm}$ measures are identical. (In particular, $P_{a}=P_{\bar{a}}$.) In addition, if $\alpha$ and $\beta$ are both adapted (resp. predictable), then it is enough for the $\mathrm{Palm}$ measures to agree on $\mathfrak{F}_{0+}^{0}$ (resp. $\left.\mathfrak{F}_{0-}^{0}\right)$.

1. Decomposition theorems and characterizations of Palm measures. A function $\xi \epsilon\left(\mathcal{F}^{0}\right)$ for which $\xi \circ \theta_{t}(\omega) \rightarrow \xi(\omega)$ as $t \downarrow 0$ for all $\omega \in \Omega$ will be called translation continuous. It is shown in [9] that there exists another filtration $\left\{\mathfrak{S}_{t}^{0}\right\}$ such that $\mathfrak{G}_{t}^{0} \subset \mathcal{F}_{t-}^{0}, \mathfrak{S}_{t}=\mathcal{F}_{t}$ for all $t \in \mathbf{R}$, where $\left\{\mathfrak{S}_{t}\right\}$ is the completed family corresponding to $\left\{\varrho_{t}^{0}\right\}$ (see $\oint_{0}$ ), and $\mathcal{G}^{0}=V_{t \in \mathrm{R}} \varrho_{t}^{0}$ is generated by the translation continuous functions. Moreover, the mapping $(t, \omega) \rightarrow \theta_{t}(\omega)$ is $\mathbb{B} \otimes \mathcal{G}^{0} / \mathcal{G}^{0}$-measurable. Since the two filtrations differ by sets of measure zero only, there is no.essential loss of generality in assuming that $\mathcal{F}^{0}$ is itself generated by the translation continuous functions. For reasons (in addition to those above) which will soon be apparent, we assume from now on 
(I) $\mathfrak{F}^{0}$ is generated by the translation continuous functions,

(II) $\left(\Omega, \mathfrak{F}^{0}\right)$ is a Blackwell space.

The meaning of (II) is: (i) $\mathcal{F}^{0}$ is separable, and (ii) for every real-valued $\xi \in\left(\mathcal{F}^{0}\right)$ and $A \in \mathcal{F}^{0}$, the image $\xi(A)$ is analytic in $\mathrm{R}$. The basic fact we will require is this: let $(E, \mathcal{G})$ be a Blackwell space and $\mathcal{G}$ a separable sub $\sigma$-field of $\mathcal{E}$. A function $f \in(\mathcal{E})$ which is constant on the atoms of $\mathcal{G}$ is

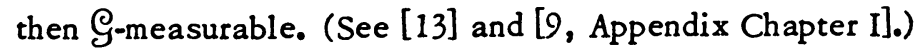

We hasten to add that many of the results below do not depend on (I), though they are more complicated without it, and that most of the standard spaces arising in flow theory satisfy (I) and (II). Two examples are the function spaces $\sqrt{ }$ and $D$ of all continuous functions (resp: right-continuous functions having left limits) from $\mathbf{R}$ to $\mathbf{R}$; for $f \in \mathcal{E}$ (or $\sqrt[9]{\text { ) }}$ we let $\theta_{t} f(s)=$ $f(s+t), X_{s} f=f(s)$, and $\mathfrak{F}_{t}^{0}=\sigma\left\{X_{s}: s \leq t\right\}$. Another example, arising in connection with point processes, is the space $\mathfrak{B}$ of all locally finite (i.e. having no finite accumlation point) nonempty subsets of R. Here, for $w \in \mathbb{B}$, we let $\theta_{t} w=w-t$ and take $W_{t}^{0}$ as the $\sigma$-field generated by the functions $N(A, w)=$ cardinality of $A \cap w$ for Borel sets $A \subset(-\infty, t]$. These examples will be discussed below.

Let $Z \in\left(\mathcal{F}_{0}\right)_{+}$be such that $Z_{t}=e^{-t} Z \circ \theta_{t}, t \in \mathbf{R}_{+}$, is a supermartingale relative to $\left\{\mathcal{F}_{t}\right\} ; Z$ is then called excessive. We need the following result, which may be derived from [8] or [9].

(5) Theorem. Let $Z$ be excessive. Then there exists an excessive $Z^{0}$ $=Z$ a.s. for which $Z^{0} \in\left(\mathcal{F}_{0_{+}}^{0}\right)_{+}$and the mapping $t \rightarrow Z^{0} \circ \theta_{t}(\omega)$ is rightcontinuous and has left limits at every $t \in \mathbf{R}$, for almost every $\omega \in \Omega$.

In particular, let $Z=\left(Z_{t}\right), t \in \mathbf{R}_{+}$, be a potential [13] such that $Z_{t}=$ $e^{-t} Z_{0} \circ \theta_{t}$ a.s. for each $t$ ( $Z$ is "almost homogeneous"). Then there exists a homogeneous potential $Z_{t}^{0}=e^{-t} Z^{0} \circ \theta_{t}$, with $Z^{0}$ as described in (5), such that $Z_{t}^{0}$ and $Z_{t}$ are indistinguishable.

Let $B_{+}$denote the Borel $\sigma$-field in $\mathbf{R}_{+}$, and define, for any process $u \epsilon$ $\left(\mathscr{B}_{+} \otimes \mathcal{F}^{0}\right)$, two new processes, $\theta^{+} u$ and $\theta^{-} u$, by

$$
\theta^{+} u(s, \omega)=u\left(s, \theta_{s} \omega\right), \quad \theta^{-} u(s, \omega)=u\left(s, \theta_{-s}(\omega) .\right.
$$

These are measurable, and $\theta^{+}$and $\theta^{-}$are obviously inverses of one another. Now define $\mathcal{P}^{0}$ to be the $\sigma$-field on $\mathbf{R}_{+} \times \Omega$ generated by all sets of the form $[t, \infty) \times A, t \in \mathbf{R}_{+}, A \in \mathcal{F}_{t-}^{0}$ (equivalently: all sets of the form $\{0\} \times A, A \in$ $\mathfrak{F}_{0-}^{0}$, and $(t, \infty) \times A, t \in \mathbf{R}_{+}, A \in \mathcal{F}_{t-}^{0}$ ). This is similar to the usual predictable $\sigma$-field [2], but more appropriate in the present context. 
(6) Lemma. $\mathscr{P}^{0}=\theta^{+}\left(\mathscr{B}_{+} \otimes \mathcal{F}_{0-}^{0}\right)$, i. e. $u \in\left(\mathcal{P}^{0}\right)$ iff $\theta^{-} u \in\left(\mathfrak{B}_{+} \otimes \mathcal{F}_{0-}^{0}\right)$.

First note that $\left(R_{+} \times \Omega, \mathscr{B}_{+} \otimes \mathcal{F}^{0}\right)$ is a Blackwell space, and $\mathscr{B}_{+} \otimes \mathcal{F}_{0-}^{0}$ is a separable sub $\sigma$-field of $\mathscr{B}_{+} \otimes \mathcal{F}^{0}$. Consider a generator of $\mathscr{P}^{0}$, say $[t, \infty) \times A$, with $A \in \mathcal{F}_{t-}^{0}$, and let $u(s, \omega)=I_{[t, \infty)}(s) I_{A}(\omega)$. We wish to show $\theta^{-} u$ is constant on the atoms of $\mathfrak{B}_{+} \otimes \mathcal{F}_{0-}^{0}$. Suppose $(s, \omega),\left(s^{\prime}, \omega^{\prime}\right)$ are contained in such an atom. Then, for every $B \in \mathfrak{B}_{+}, C \in \mathfrak{F}_{0_{-}}^{0}$, we have $I_{B}(s) I_{C}(\omega)=I_{B}\left(s^{\prime}\right) I_{C}\left(\omega^{\prime}\right)$, so $s=s^{\prime}$ and $\omega, \omega^{\prime}$ lie in the same atom of $\mathcal{F}_{0-}^{0}$. Hence $\theta^{-} u(s, \omega)=I_{[t, \infty)}(s) I_{A}\left(\theta_{-s} \omega\right)$ and $\theta^{-} u\left(s^{\prime}, \omega^{\prime}\right)=I_{[t, \infty)}(s) I_{A}\left(\theta_{-s} \omega^{\prime}\right)$ both vanish if $s \leq t$, and are equal if $s \geq t$, because then $\theta_{s} A \in \mathcal{F}_{(t-s)-}^{0} C$ $\mathfrak{F}_{0-}^{0}$. We have shown $\mathcal{P}^{0} \subset \theta^{+}\left(\mathscr{B}_{+} \otimes \mathcal{F}_{0_{-}}^{0}\right)$. Next, let $v \in\left(\mathscr{B}_{+} \otimes \mathcal{F}_{0_{-}}^{0}\right)$ be of the form $v(s, \omega)=I_{B}(s) I_{C}(\omega), B \in \mathfrak{B}_{+}, C \in \mathcal{F}_{0-}^{0}$. Noting that $\mathcal{P}^{0}$ is a separable sub $\sigma$-field of $B_{+} \otimes \mathcal{F}^{0}$, we will show that $\theta^{+} v$ is constant on atoms of $\rho^{0}$. Let $(s, \omega),\left(s^{\prime}, \omega^{\prime}\right)$ be in an atom of $\mathcal{P}^{0}$. Then $s=s^{\prime}$, and $I_{A}(\omega)=$ $I_{A}\left(\omega^{\prime}\right)$ for every $A \in \mathcal{F}_{s-}^{0}$. Thus $v\left(s, \theta_{s} \omega\right)=I_{B}(s) I_{C}\left(\theta_{s} \omega\right)=I_{B}\left(s^{\prime}\right) I_{C}\left(\theta_{s} \omega\right)$ since $\theta_{s}^{-1} C \in \mathcal{F}_{s-}^{0}$. The proof is complete.

(7) Corollary. (a) If $t \in \mathbf{R}_{+}$and $A \in \mathcal{F}_{t+}^{0}$, then $(t, \infty) \times A \in \mathcal{P}^{0}$. (b) If $\xi \in\left(\mathcal{F}_{0_{-}}^{0}\right)$, then the process $\xi \circ \theta=\left(\xi \circ \theta_{t}(\omega)\right)$ is $\mathcal{P}^{0}-$ measurable. Part (a) results from $(t, \infty) \times A=\bigcup_{n=1}^{\infty}\left(t+n^{-1}, \infty\right) \times A$; (b) is trivial. To each measure $Q$ on $\mathcal{F}_{0-}^{0}$ we now associate a measure $\tilde{Q}$ on $\mathcal{P O}^{0}$ as follows. Writing $\widetilde{Q}(u)$ for $\int u d \widetilde{Q}$,

$$
\tilde{Q}(u)=\int_{0}^{\infty} e^{-s} \int_{\Omega} \theta^{-} u(s, \omega) Q(d \omega) d s, \quad u \in\left(\mathscr{P}^{0}\right)_{+} .
$$

We further write $\tilde{Q}_{t}(A)=\tilde{Q}[(t, \infty) \times A], A \in \mathcal{F}_{t+}^{0}$, which is possible by (7)(a), and call $\tilde{Q}$ (or $Q$ ) progressively absolutely continuous (relative to $P$ ) if, for each $t \in \mathbf{R}_{+}, \tilde{Q}_{t} \ll P$ on $\mathcal{F}_{t+}^{0}$, or, equivalently, $\tilde{Q}_{0} \ll P$ on $\mathcal{F}_{0_{+}}^{0}$

(9) Theorem. A finite measure $Q$ on $\mathfrak{F}_{0-}^{0}$ which is progressively absolutely continuous may be written uniquely as $Q=P_{\alpha}^{-}+\mu$ where $P_{\alpha}^{-}$is the restriction to $\mathcal{F}_{0_{-}}^{0}$ of the Palm measure of an (integrable) predictable AF a, and the measure $\mu$ is concentrated on a polar set.

The meaning of "polar" is this. Let $\xi$ be a random variable on $\Omega$; the

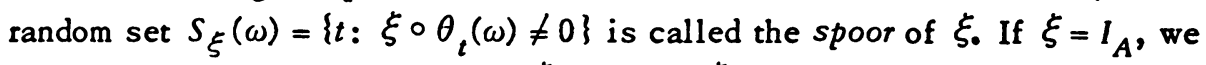
speak of the "spoor of $A$ ". Then $\xi$ (or $A$ if $\xi=I_{A}$ ) is called polar (thin) if its spoor is a.s. empty (a.s. locally finite). Thus $\xi$ is polar iff $\xi \circ \theta=$ $\left(\xi \circ \theta_{t}(\omega)\right)$ is an "evanescent" process [2]. Further, $A$ is semipolar if it is contained in a countable union of thin sets. As indicated earlier, $P_{a}^{-}$further decomposes into a measure which charges no semipolar and a measure which 
lives on a semipolar, but charges no polar, set (see $\$ 2$ ). We note that if $Q$ charges no polar set in $\mathcal{F}_{0-}^{0}$, then (using a Fubini argument) it is progressively absolutely continuous.

As an immediate consequence of (9) (using the trivial filtration $\mathcal{F}_{t}^{0} \equiv \mathfrak{F}^{0}$ for (b)) we have

(10) Theorem. (a) A finite measure $Q$ on $\mathfrak{F}_{0-}^{0}$ is the restriction to $\mathfrak{F}_{0-}^{0}$ of the Palm measure of an integrable, predictable AF iff $Q$ charges no polar set in $\mathfrak{F}_{0-}^{0}$.

(b) A finite measure $Q$ on $\mathcal{F}^{0}$ is a Palm measure iff $Q$ charges no polar set in $\mathcal{F}^{0}$, in which case the corresponding $A F\left(\alpha_{t}\right)$ is the right-continuous regularization of $\left(d Q^{t} / d P\right)$ where $Q^{t}(A)=\int_{0}^{t} Q\left(\theta_{s} A\right) d s, A \in \mathcal{F}^{0}$.

Proof of (9). The uniqueness is clear, since (3) implies that a Palm measure charges no polar set. Let $Z_{t} \in\left(\mathcal{F}_{t_{+}}^{0}\right)_{+}$be the Radon-Nikodym derivative $d \tilde{Q}_{t} / d P$ on $\mathcal{F}_{t+}^{0}$. Since $t \rightarrow E Z_{t}$ is right-continuous one may choose an a.s. right-continuous version $Z=\left(Z_{t}\right)$ and it is easy to see that $Z$ is a potential, though not necessarily of class $(D)$ (see [13] for terminology).

Now a change of variables yields $\widetilde{Q}_{t}(A)=e^{-t} \widetilde{Q}_{0}\left(\theta_{t} A\right), A \in \mathcal{F}_{t+}^{0}$, from which follows immediately that, for each $t \in \mathbf{R}_{+}, Z_{t}=e^{-t} Z_{0} \circ \theta_{t}$ a.s. By the remark following (5), we may assume $Z_{t}$ is homogeneous: $Z_{t} \equiv$ $e^{-t} Z_{0} \circ \theta_{t}$.

It is well known that any potential $Z$ has a unique decomposition $Z=$ $N+Y$, where $N$ is a local martingale (and also a potential) and $Y$ is a potential of class $(D)$. We show now that $N$ and $Y$ may be chosen homogeneous. Notice that $Z_{t+s}(\omega)=e^{-t} Z_{s} \circ \theta_{t}(\omega)$ for all $\omega, s, t$. For $t$ fixed, consider the following two decompositions of $Z_{t+s}, s \geq 0$.

$$
Z_{t+s}=\left\{\begin{array}{l}
N_{t+s}+Y_{t+s}, \\
e^{-t} N_{s} \circ \theta_{t}+e^{-t} Y_{s} \circ \theta_{t} .
\end{array}\right.
$$

It is tedious, but straightforward, to check that both $N_{t+s}$ and $e^{-t} N_{s} \circ \theta_{t}$, $s \geq 0$, are local martingales and that both $Y_{t+s}$ and $e^{-t} Y_{s} \circ \theta_{t}, s \geq 0$, are class (D) potentials, all relative to the $\sigma$-fields $\mathcal{F}_{t+s}, s \geq 0$. By uniqueness, the two expressions "match" correctly, and we conclude $N_{t+s}=$ $e^{-t} N_{s} \circ \theta_{t}$ for all $s$, $a_{0} s_{0}$, and similarly for $Y_{t+s^{\circ}}$ Putting $s=0$, we find $N, Y$ are "almost homogeneous" and may be replaced by homogeneous modifications, which we again denote by $N$ and $Y$.

By the Doob-Meyer decomposition theorem [13, p. 119] we may write $Y=$ $M-A$, where $M=\left(M_{t}\right)$ is a uniformly integrable martingale, and $A=\left(A_{t}\right)$ 
is a predictable (= natural) integrable increasing process. Since $Y$ is homogeneous, we have two decompositions of $Y_{t+s}$ for $s \geq 0$ ( $t$ fixed) analogous to (11):

$$
Y_{t+s}=\left\{\begin{array}{l}
M_{t+s}-A_{t+s}=M_{t+s}-A_{t}-\left(A_{t+s}-A_{t}\right), \\
e^{-t} M_{s} \circ \theta_{t}-e^{-t} A_{s} \circ \theta_{t} .
\end{array}\right.
$$

Noting that $e^{-t} M_{s} \circ \theta_{t}$ and $M_{t+s}-A_{t}$ are uniformly integrable martingales, and $e^{-t} A_{s} \circ \theta_{t}, A_{t+s}-A_{t}$ are predictable increasing processes (all relative to $\left\{\mathcal{F}_{t+s}\right\}, s \geq 0, t$ fixed), we have by the uniqueness of the decomposition,

$$
A_{t+s}-A_{t}=e^{-t} A_{s} \circ \theta_{t}
$$

Now let $a_{t}=\int_{0}^{t} e^{s} d A_{s}$. (By $\int_{a}^{b}$ we will always mean $\int_{(a, b]}$.) From (12) it follows easily that $\alpha$ is a predictable AF. (This argument was inspired by Maisonneuve [10].)

Now for any Palm measure, say $P_{\beta}$, we have (see [5])

(13) $E \int_{\mathbf{R}} u(s, \omega) \beta(d s, \omega)=\int_{\Omega} \int_{\mathbf{R}} u\left(s, \theta_{-s} \omega\right) d s P_{\beta}(d \omega), \quad u \in\left(\mathbb{B} \otimes \mathcal{F}^{0}\right)_{+} \cdot$ Hence for any $A \in \mathcal{F}_{t+}^{0}$,

$$
E\left(Y_{i} ; A\right)=E\left(\int_{t}^{\infty} e^{-s} d \alpha_{s} ; A\right)=\int_{t}^{\infty} e^{-s} P_{\alpha}\left(\theta_{s} A\right) d s .
$$

So we may write

$$
\text { (14) } \int_{t}^{\infty} e^{-s} Q \circ \theta_{s}(A) d s=E\left(N_{t} ; A\right)+\int_{t}^{\infty} e^{-s} P_{a} \circ \theta_{s}(A) d s, \quad A \in \mathcal{F}_{t^{+}}^{0} \text {. }
$$

Now define a measure $\tilde{\mu}$ on $\mathcal{P}^{0}$ by equation (8) with $Q$ replaced by $\mu=$ $Q-P_{a}^{-}, P_{a}^{-}$being the restriction of $P_{a}$ to $\mathcal{F}_{0-}^{0}$. Using (14), we see that $\tilde{\mu}$ is positive on sets of the form $(t, \infty) \times A, A \in \mathcal{F}_{t+}^{0}$, and hence on all of $\rho^{0}$; moreover

$$
E\left(N_{t} ; A\right)=\tilde{\mu}[(t, \infty) \times A]=\int_{t}^{\infty} e^{-s} \mu \circ \theta_{s}(A) d s, \quad A \in \mathcal{F}_{t^{+}}^{0} .
$$

(The existence of a measure $\tilde{\mu}$ on $R_{+} \times \Omega$ satisfying the first equality in (15) is established by Föllmer [3] in a different situation.) It is easy to see that $\mu \geq 0$ so it only reamins to prove that $\mu$ lives on a polar set.

Having chosen $N$ homogeneous, i.e. $N_{t} \equiv e^{-t} \bar{n} \circ \theta_{t}$ for an excessive function $\bar{n} \in\left(\mathcal{F}_{0_{+}}^{0}\right)_{+}$, note first that we may extend $N_{t}$ to all $t \in \mathbf{R}$ and still have a supermartingale. Moreover, $N_{t}$ will be right-continuous with finite left limits for all $t \in \mathbf{R}$ a.s. since $t \rightarrow \bar{n} \circ \theta_{t}$ has these properties. 
Define, for each $n \geq 1, R_{n}=\inf \left\{r>0 \text {, rational: } N_{r}>n\right\}_{\text {. Each }} R_{n} \in \mathcal{S}_{+}^{0}$, the family of stopping times of $\left\{\mathcal{F}_{t+}^{0}\right\}, t \in \mathbf{R}_{+}$, and on right-continuous paths coincides with $\inf \left\{r>0: N_{r}>n\right\}$. Starting with discrete $T \in \mathcal{S}_{+}^{0}$, one easily shows that the stochastic interval $]] T, \infty\left[\left[\in \mathcal{P}^{0}\right.\right.$ for any $T \in \mathcal{S}_{+}^{0}$, and hence $\left.\left.K=\bigcap_{n}\right]\right] R_{n}, \infty\left[\left[\in \mathcal{P}^{0}\right.\right.$. The argument in [3] shows that $\tilde{\mu}$ is supported by $K$ and that $K$ is evanescent. (The set $K$ in [3] is slightly different, but, since $\tilde{\mu}$ puts no mass on $\{\infty\} \times \Omega$ because $N_{t}$ is a potential, the argument given there goes through.)

Define $\xi(\omega)=\int_{0}^{\infty} e^{-r} I_{K}\left(r, \theta_{-r} \omega\right) d r$; since $K \in \mathscr{P}^{0},(6)$ implies $\xi \in\left(\mathcal{F}_{0-}^{0}\right)$. Clearly $\mu(\xi)=\tilde{\mu}(K)$, and $\mu(1-\xi)=\tilde{\mu}(K)=0$. Hence $\xi=1 \mu$-a.e. and $\mu\{\xi=0\}=0$, i.e. $\mu$ lives on the set $\{\xi>0\}$. To show this set is polar, let $G$ be the set of $\omega \in \Omega$ on which the trajectory $N_{t}(\omega)$ has finite left limits and is right-continuous at all $t \in \mathbf{R}$. Then $G$, and so $G^{c}$, is invariant, and $P\left(G^{c}\right)=0$. Suppose $\xi(\omega)>0$. Then $\left(r, \theta_{-r} \omega\right) \in K$ for some $r>0$, i.e. $R_{n}\left(\theta_{-r} \omega\right)<r$ for all $n \geq 1$, and this puts $\omega$ in $G^{c}$. But $\{\xi>0\} \subset G^{c}$ implies $\{\xi>0\}$ is polar since $G^{c}$ is an invariant null set. This completes the proof of (9).

We now sketch the proof of another decomposition for an arbitrary (finite) $Q$ on $\mathcal{F}_{0-}^{0}$, which is valid under the additional assumption that $\left\{\mathcal{F}_{t}^{0}\right\}$ is a standard system [3], [15], which means

(a) each $\mathcal{F}_{t}^{0}$ is $\sigma$-isomorphic to the Borel $\sigma$-field of a Polish space;

(b) for any increasing sequence $t_{n}$, and decreasing sequence of sets $A_{n}$, such that $A_{n}$ is an atom of $\mathcal{F}_{t_{n}}^{0}$, we have $\bigcap_{n} A_{n} \neq \varnothing$.

Unfortunately, the usual filtrations on the standard spaces of flow theory, such as $\sqrt{ }$ and $\mathfrak{B}$ previously mentioned, are not standard in the above sense. We will indicate later how to circumvent this difficulty for those two cases.

Let $Q$ be a finite measure on $\mathcal{F}_{0}^{0}$ and define $\widetilde{Q}$ as before (see (8)). For each $t \in \mathbf{R}_{+}$, the measure $\tilde{Q}_{t}$ has a Lebesgue decomposition on $\mathcal{F}_{t+}^{0}$, namely $\tilde{Q}_{t}(A)=Q_{t}^{\prime}(A)+Q_{t}^{\prime \prime}(A)$, with $Q_{t}^{\prime} \ll P$ on $\mathcal{F}_{t+}^{0}$, and $Q_{t}^{\prime \prime} \perp P$ on $\mathcal{F}_{t+}^{0}$ ( 1 means "singular"). An easy argument using the uniqueness of the Lebesgue decomposition shows that $Q_{t}^{\prime}=e^{-t} Q_{0}^{\prime} \circ \theta_{t}$ and $Q_{t}^{\prime \prime}=e^{-t} Q_{0}^{\prime \prime} \circ \theta_{t}$. Let $Z_{t}=d Q_{t}^{\prime} / d P$ on $\mathcal{F}_{t+}^{0}$. We may choose a homogeneous version of the potential $Z=\left(Z_{t}\right)$ just as in the proof of (9), and this splits into a local martingale $N$ plus a class $(D)$ potential $Y$, both of which are homogeneous. Now let $\tilde{\mu}$ be the Föllmer measure of the local martingale $N$, i.e. the unique measure on $P 0$ such that the first equality in (15) holds. We thus have, $a$ being as in the proof of (9),

$$
\widetilde{Q}=\tilde{Q}_{\alpha}+\tilde{\mu}+\tilde{\nu}
$$


where $\widetilde{Q}_{a}$ is defined by the right-hand side of (8) with $P_{a}$ in place of $Q, \tilde{\nu} \equiv$ $\widetilde{Q}-\widetilde{Q}_{a}-\tilde{\mu}$. In this way, $\widetilde{\nu}[(t, \infty) \times A]=Q_{t}^{\prime \prime}(A), A \in \mathcal{F}_{t+}^{0}$.

Equation (16) exhibits $\widetilde{Q}$ as the sum of the progressively absolutely continuous measure $\widetilde{M}=\widetilde{Q}_{\alpha}+\tilde{\mu}$ and the "progressively singular" measure $\tilde{\nu}$. One establishes easily that such a decomposition is unique.

Define measures $\mu, \nu$ on $\mathcal{F}_{0-}^{0}$ by $\mu(A)=\tilde{\mu}\left(I_{A} \circ \theta\right), \nu(A)=\widetilde{\nu}\left(I_{A} \circ \theta\right)$.

(17) Lemma. For every $u \in\left(\rho^{0}\right)_{+}$,

$$
\tilde{\mu}(u)=\int_{\Omega} \int_{0}^{\infty} e^{-s} \theta-u(s, \omega) d s \mu(d \omega)
$$

and similarly for $\tilde{\nu}$ and $\nu$.

Proof. Define a transformation $T_{t}$ on $\mathfrak{B}_{+} \otimes \mathcal{F}^{0}$ for each $t \in \mathbf{R}_{+}$by $T_{t} u(s, \omega)=u\left(s+t, \theta_{-t} \omega\right)$. A monotone class argument shows that $T_{t}:\left(\mathcal{P}^{0}\right)_{+}$ $\rightarrow\left(\mathcal{P}^{0}\right)_{+}$. From $(8)$ we find that

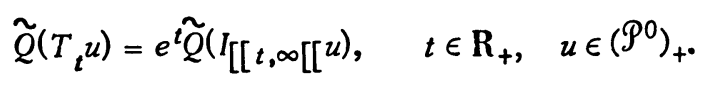

By looking at the generators of $\mathcal{P}^{0}$, we also find that $\tilde{M}\left(T_{t} u\right)$ defines a progressively absolutely continuous measure, while $\widetilde{\nu}\left(T_{t} u\right)$ is progressively singular ( $t$ fixed). The uniqueness of the decomposition (16) shows that (19) holds for $\widetilde{M}$ (resp. $\widetilde{\nu}$ ) in place of $\widetilde{Q}$; since $\widetilde{Q}_{a}$ also satisfies (19), so does $\tilde{\mu}$. In view of (6), it will suffice to verify (18) for $u=\theta^{+}\left(I_{[t, \infty)} \xi\right), t \geq 0, \xi \epsilon$ $\left(\mathcal{F}_{0_{-}}^{0}\right)_{+}$, in which case the right-hand side of $(18)$ reduces to $e^{-t} \tilde{\mu}(\xi \circ \theta)$. The left-hand side is

$$
\begin{aligned}
\tilde{\mu}\left(I_{[[t, \infty[[} \theta^{+} \xi\right) & =e^{-\tau} \tilde{\mu}\left(T_{t}(\xi \circ \theta)\right) \\
& =e^{-\tau} \tilde{\mu}(\xi \circ \theta) .
\end{aligned}
$$

The fact that $\mu$ is carried by a polar set is proven just as before and we can state, given that $\left\{\mathcal{F}_{t}^{0}\right\}$ is standard:

(20) Theorem. A finite measure $Q$ on $\mathfrak{F}_{0-}^{0}$ may be written as $Q=P_{\bar{a}}^{-}+$ $\mu+\nu$, where $P_{a}^{-}$and $\mu$ are as described in (9), and $\nu$ is such that

$$
\tilde{\nu}(u)=\int_{\Omega} \int_{0}^{\infty} e^{-s} \theta^{-} u(s, \omega) d s \nu(d \omega), \quad u \in\left(\mathcal{P}^{0}\right)_{+},
$$

is progressively singular.

As we indicated, the spaces $\mathbb{C}, \mathscr{D}$ and $\mathfrak{B}$ are not standard. To illustrate how to overcome this difficulty, introduce the space $\mathcal{C}^{\prime}$ consisting of all functions $f: \mathbf{R} \rightarrow \mathbf{R} \cup\{\Delta\}$, where $\Delta \notin \mathbf{R}$ is an adjoined "death point" such that $f$ is continuous on $\mathbf{R}$ for all $t<\zeta$ and $f(t)=\Delta$ for all $t \geq \zeta$. The "lifetime" $\zeta \leq \infty$ depends on $f$. Clearly $\subseteq \subset \mathcal{C}^{\prime}$, and $f \in \mathcal{C}$ iff $\zeta=+\infty$. Again let 
$\theta_{t}$ "shift" $f$ by $t, X_{t} f=f(t)$, and define $\mathcal{F}_{t}^{\prime}=\sigma\left\{X_{s}, s \leq t\right\}$, all relative to $\mathfrak{S}^{\prime}$. In this way, $\mathcal{F}_{t}^{0}=\mathcal{F}_{t}^{\prime} \cap \mathbb{E}, \mathbb{E}$ is an invariant subset of $\mathbb{E}^{\prime}$, and a stationary measure $P$ on $\mathcal{C}$ extends naturally to $\mathcal{C}^{\prime}$ by $P^{\prime}(A)=P(A \cap \mathbb{E}), A \in \mathcal{F}^{\prime}=$ $\bigvee_{t} \mathcal{F}_{t}^{\prime}$. The filtration $\left\{\mathcal{F}_{t}^{\prime}\right\}$ is standard. (To "standardize" $\mathbb{B}$, one introduces $\mathbb{B}^{\prime}$ ' consisting of all nonempty subset's $w \in \mathbf{R}$ which are locally finite before $\zeta=\sup w \leq \infty$. We only pursue the case $C^{\prime}$; the argument for $\mathbb{B}$ is entirely similar.)

Let $Q$ be a finite measure on $\mathcal{F}_{0_{-}}^{0}=S_{S} \cap \mathcal{F}_{0_{-}}^{\prime}$, and extend it to $\mathcal{F}_{0_{-}}^{\prime}$ by $Q^{\prime}(A)=Q\left(A^{\prime} \mathbb{E}\right), A \in \mathcal{F}_{0-}^{\prime}$. By (20)

$$
Q^{\prime}=P_{a}^{-}+\mu+\nu,
$$

all measures on $\mathcal{F}_{0_{-}}^{\prime}$. Restricting each of these to $\mathcal{F}_{0_{-}}^{0} \subset \mathcal{F}_{0_{-}}^{\prime}$ - we obtain a decomposition of $Q$; it only remains to show it is of the desired form. Now $P_{a}^{-}$kills every polar set in $\mathcal{F}_{0}^{0}$ since every polar set in $\mathcal{F}^{0}$ relative to $P$ is also polar relative to $P^{\prime}$; indeed, the restriction to $C$ of $P_{a}$ is the $\mathrm{Palm}$ measure of the restriction of $a$ to $\mathfrak{E}$. As for the restriction to $\mathcal{F}_{0-}^{0}$ of $\mu$, it is obvious that it lives on $N\left(\mathcal{S}\right.$, where $N \in \mathfrak{F}_{0-}^{\prime}$ is polar and $\mu\left(N^{C}\right)=0$, and that $N \subseteq \in \mathcal{F}_{0_{-}}^{0}$ is polar. One also can show that $\nu$ restricted to $\mathcal{F}_{0-}^{0}$ is as in (20).

Finally we remark that, if we write the Lebesgue and $\tilde{Q}_{t}^{\prime}\left(A^{\prime}\right)$ (with the obvious notation),

$$
\begin{aligned}
& \tilde{Q}_{t}(A)=E\left(Z_{t} ; A\right)+K_{t}(A), \quad A \in \mathcal{F}_{t^{+}}^{0}, \\
& \tilde{Q}_{t}^{\prime}\left(A^{\prime}\right)=E^{\prime}\left(Z_{t}^{\prime} ; A^{\prime}\right)+\mathrm{K}_{t}^{\prime}\left(A^{\prime}\right), \quad A^{\prime} \in \mathcal{F}_{t^{\prime}}^{\prime},
\end{aligned}
$$

where $K_{t}, K_{t}^{\prime}$ are $P$-singular (resp. $P^{\prime}$-singular), and $Z_{t}, Z_{t}^{\prime}$ are the RadonNikodym derivatives of the $P-\left(P^{\prime}-\right)$ absloutely continuous pieces, then $Z$, $Z^{\prime}$ are potentials and the pieces match properly. Indeed, $K_{t}^{\prime}\left(A^{\prime}\right)=K_{t}\left(A^{\prime} \mathbb{E}\right)$, and $Z_{t}^{\prime}$ may be taken as the "canonical extension" of $Z_{t}$ to $\mathcal{C}^{\prime}$ : first choose the homogeneous version of $Z$, and then let $Z_{t}^{\prime}(f)=0$ if $t \geq \zeta$ and $Z_{t}^{\prime}(f)=$ $Z_{t}(\tilde{f})$ if $t<\zeta$, where $\tilde{f} \in \mathcal{S}$ is any function agreeing with $f \in \mathcal{S}^{\prime}$ for all $s \leq t+\epsilon$ for sufficiently small $\epsilon>0$. The $\mathcal{F}_{t}^{0}$-measurability of $Z_{t}$ guarantees the definition to be independent of the choice of $\tilde{f}$, and $Z^{\prime}$ is also homogeneous.

It is an open question whether every filtered dynamical system can be embedded in a standard system as in the above examples. We should also mention that the measure $\nu$ in (20) is something of a mystery to us.

We conclude this section by pointing out two applications of our results. Let $A^{\circ}=\left(A_{t}\right), t \in \mathbf{R}_{+}$, be an integrable, increasing process relative to $\left\{\mathcal{F}_{t}\right\}$, that is (see [2]), $A_{0} \equiv 0, A$ is right-continuous, nondecreasing and $E A_{\infty}<\infty$. 
Define a measure $\mu_{A}$ on $R_{+} \times \Omega$ by

$$
\mu_{A}(u)=E \int_{0}^{\infty} u(s, \omega) d A_{s}(\omega), \quad u \in\left(B_{+} \otimes \mathcal{F}^{0}\right)_{+},
$$

and a (finite) measure $Q$ on $\mathscr{F}^{0}$ by $Q(\xi)=\mu_{A}(\xi \circ \theta)$. Obviously, $Q$ kills every polar set in $\mathcal{F}^{0}$, and hence by $(10)$ is the Palm measure of an (integrable) AF $\alpha$. One easily checks that, in fact,

$$
a_{t}(\omega)=\int_{\mathbb{R}} \int_{0}^{\infty} I_{(0, t]}(s+r) d A_{r}\left(\theta_{s} \omega\right) d s, \quad t \in \mathbf{R}_{+} \cdot
$$

We call $a$ the additive projection of $A$. A special case of this is used in [6], and Mecke [12] has noted a similar idea.

The following result has a Markovian analogue [16].

(22) Theorem. A set $N \in \mathcal{F}^{0}$ is polar if and only if it is charged by no Palm measure.

Proof. If $N$ is polar, we have already observed that $P_{a}(N)=0$ for every AF $\alpha$. Suppose $N$ is not polar. Its spoor $S_{N}(\omega)$ is then nonempty on a set of $\omega$ having positive probability; in fact, for almost every $\omega \in \Omega$ for which $S_{N}(\omega) \neq \varnothing$, we shall see that $S_{N}(\omega)$ is unbounded above. Observe, to begin with, that $S_{N}(\omega)$ is a "homogeneous sei" in the sense that for every $t \in \mathbf{R}$, $S_{N}\left(\theta_{t} \omega\right)=S_{N}(\omega)-t$. Let $B_{N}=\left\{\sup S_{N}>n\right\}$. Since the projection on $\Omega$ of sets in $\mathfrak{B} \otimes \mathcal{F}$ is in $\mathcal{F}$ (see [2, I., T32]), $B_{n} \in \mathcal{F}$ for each $n \geq 1$. The ergodic theorem now yields

$$
\lim _{t \rightarrow \infty} t^{-1} \int_{0}^{t} I_{B_{n}}\left(\theta_{-s} \omega\right) d s=P\left(B_{n} \mid \mathscr{Q}\right) \text { a.s. }
$$

where $\mathcal{A}$ denotes the $\theta$-invariant $\sigma$-field in $\Omega$. Suppose $S_{N}(\omega) \neq \varnothing$. Then $S_{N}\left(\theta_{-s} \omega\right)=S_{N}(\omega)+s$ has supremum greater than $n$ when $s$ is sufficiently large. Hence if $B=\left\{S_{N} \neq \varnothing\right\}$, we have $P\left(B_{n} \mid \mathfrak{Q}\right)=1$ a.s. on $B$. Since $B$ is invariant, $P\left(B \cap B_{n}^{c}\right)=0$, which proves our point.

Now set $D(\omega)=S_{N}(\omega) \cap(0, \infty) \in \Re_{+}$; clearly $P(D \neq \varnothing)=P(B)>0$. According to [2, I., T37], there exists a nonnegative random variable $\tau \in(\mathcal{F})_{+}$ such that $r(\omega) \in D(\omega)$ for $D(\omega) \neq \varnothing$ and otherwise $r=\infty$. Define an integrable, increasing process $A=\left(A_{t}\right)$ by $\left.A_{t}(\omega)=I_{\{\tau \leq t}\right\}(\omega) ; A$ is flat, except for $a$ unit jump at $r$ if $r<\infty$. We then have

$$
\mu_{A}\left(I_{N} \circ \theta\right)=E \int_{0}^{\infty} I_{N} \circ \theta_{s}(\omega) d A_{s}(\omega)=E\left(I_{N} \circ \theta_{\tau} ; D \neq \varnothing\right)=P(B)>0 .
$$

By our work above, $P_{a}(N)=\mu_{A}\left(I_{N} \circ \theta\right)>0$, where $\alpha$ is the additive projection of $A$, and (22) is proven.

Note. A similar argument, using the material in [2, Chapter VI (esp. $\$ 3$ and T37)], shows that a set $N \in \mathcal{F}^{0}$ has an a.s. countable spoor $S_{N}(\omega)$ iff $P_{a}(N)=0$ for every continuous AF $\alpha$. 
For our second application, let $X=\left(X_{t}\right), t \in \mathbf{R}$, be a strictly stationary measurable process such that $X_{t}=X_{0} \circ \theta_{t}$, and assume $X$ is adapted to a filtration $\left\{\mathcal{F}_{t}^{0}\right\}$. Denote by $(E, \mathcal{E})$ the state space of $X$, with $\mathcal{E}$ assumed separable, and let $\pi(\Gamma)=P\left\{X_{t} \in \Gamma\right\}$ (independent of $t$ ) be the one-dimensional distribution of the process. We say that $X$ has a local time if there exist AF's $\alpha^{x}=\left(\alpha_{t}^{x}\right), x \in E$, such that, for almost every $\omega \in \Omega$,

$$
\int_{\Gamma} \alpha_{t}^{x}(\omega) \pi(d x)=\int_{0}^{t} I_{\Gamma}\left(X_{s}(\omega)\right) d s \text { for all } t \in \mathbf{R}_{+}, \Gamma \in \mathcal{E} \text {. }
$$

(Suitable measurability restrictions must be imposed; we omit the details.) Let $\left\{P^{x}\right\}, x \in E$, be a regular conditional probability given $X_{0}$, i.e. a family of measures on $\mathcal{F}^{0}$ such that, for every $A \in \mathcal{F}^{0}, \Gamma \in \mathcal{E}, P\left(A, X_{0} \in \Gamma\right)=$ $\int_{\mathbf{T}} P^{x}(A) \pi(d x)$. We know [4] a local time exists iff $P^{x}$ is a Palm measure for $\pi$-a.e. $x \in E$ (in which case the AF's are predictable for a.e. $x$ ).

Suppose only that there exist AF's $\alpha^{x}$ which are predictable and such that $P^{x}=P_{a^{x}}$ on $\mathcal{F}_{0-}^{0}$ for a.e. $x$. Set

$$
\beta_{t}^{\Gamma}(\omega)=\int_{0}^{t} L_{\Gamma}\left(X_{s}(\omega)\right) d s, \quad \alpha_{t}^{\Gamma}(\omega)=\int_{\Gamma} \alpha_{t}^{x}(\omega)_{\pi}(d x),
$$

for $t \in \mathbf{R}_{+}, \Gamma \in \mathcal{E}$. An easy computation shows that $\beta^{\Gamma}$ and $\alpha^{\Gamma}$ have the same Palm measure on $\mathfrak{F}_{0-}^{0}$, hence on all of $\mathcal{F}^{0}$ since $\beta^{\mathbf{\Gamma}}$ and $\alpha^{\mathbf{\Gamma}}$ are both predictable. Consequently $\alpha_{t}^{\mathbf{\Gamma}}(\omega)=\beta_{t}^{\mathbf{r}}(\omega)$ for all $t$, a.s., which yields (using separability of $\varepsilon$ ) $\alpha_{t}^{\mathbf{\Gamma}}(\omega)=\beta_{t}^{\Gamma}(\omega)$ for all $t$ and all $\Gamma$, a.s. Thus we have proven

(24) Theorem. A local time exists iff, for almost every $x \in E$, the measure $P^{x}$ charges no polar set in $\mathcal{F}_{0-}^{0}$.

Notice, however, that "polar" is defined in terms of sets in $\Omega$ which are avoided by the flow $\theta_{t}$ rather than those in $E$ which are avoided by the process $X_{t}$.

We conclude with an example, based on a construction due to Maisonneuve [10], of the "local time" of an arbitrary random set. Suppose, for each $\omega \in \Omega$, we are given a Borel set $M(\omega)$ of $\mathbf{R}$, homogeneous in that $M\left(\theta_{t} \omega\right)=M(\omega)-t$ for all $t \in \mathbf{R}, \omega \in \Omega$. (For example, $M(\omega)=\left\{t: X_{t}(\omega)=0\right\}$ with $\left(X_{t}\right)$ as above.) Define $r=\inf \left(M \cap(0, \infty)\right.$ ) (or $r=\infty$ if $M \cap(0, \infty)$ is empty), and $\tau_{t}(\omega)=t+$ $r \circ \theta_{t}(\omega)$. The random variable $\tau$ is "terminal": $r=\tau_{t}$ whenever $r>t$.

Let $Z=E\left(e^{-\tau} \mid \mathcal{F}_{0}\right)$. Then $Z$ is excessive, and by (5) we may choose a nice version of $Z$ so that $Z_{t}=e^{-t} Z \circ \theta_{t}$ is a homogeneous potential, obviously of class $(D)$. Proceeding as in the proof of (9) we write the decomposition $Z_{t}=M_{t}-A_{t}$, and let $\alpha_{t}=\int_{0}^{t} e^{s} d A_{s}$. Then $\alpha$ is a predictable AF. Under further conditions on $M, a(d t, \omega)$ is a.s. carried by $M(\omega)$. The Palm measure of $a$ is 


$$
E_{a}(\xi)=-E \int_{0}^{\infty} \xi^{*} \circ \theta_{s} d e^{-\tau_{s}}, \quad \xi \in\left(\mathcal{F}^{0}\right)_{+},
$$

where $\xi^{*}$ is the "predictable projection" of $\xi$. Put another way, $a$ is just the predictable projection of $-e^{t} d e^{-\tau}$ (see $\$ 2$ ). If $X=\left(X_{t}\right)$ has a local time, say $a^{x}$, we do not know the connection, if any, between $\alpha^{x}$ and the local time of $M^{x}(\omega)=\left\{t: X_{t}(\omega)=x\right\}, x \in E$, at least outside the Markov case.

2. Characterization of additive functionals. With assumptions (I), (II) of $\$ 1$ still in force, we will need the following basic fact, borrowed from [8] (see also [9]).

(25) Theorem. For every $\xi \in\left(\mathcal{F}^{\circ}\right)$ which is either bounded or nonnegative, there exists $\xi^{\#} \in\left(\mathcal{F}_{0_{+}}^{0}\right)\left(\right.$ resp. $\left.\xi^{*} \in\left(\mathcal{F}_{0-}^{0}\right)\right)$ such that $\left(\xi^{\#} \circ \theta_{t}\right), t \in \mathbf{R}\left(\right.$ resp. $\left.\left(\xi^{*} \circ \theta_{t}\right)\right)$ is the well-measurable (resp. predictable) projection of the process $\left(\xi \circ \theta_{t}\right), t \in \mathbf{R}$.

Moreover, $\xi^{\sharp}$ (resp. $\xi^{*}$ ) is bounded or nonnegative with $\xi$ and is unique up to a polar function. We note that the process $\xi^{*} \circ \theta$ is actually in $\left(\mathcal{P}^{0}\right)$ by (7)(b) while the notions of well-measurability, etc. refer to the family $\left\{\mathcal{F}_{t}\right\}$.

Our results in this section will be of two kinds: the first type classifies an AF $\alpha$ in accordance with the behavior of $P_{a}$ under projection, while, in the second type, we give conditions under which, for example, the dual predictable projection of $a$ is a.s. absolutely continuous. These latter results are generalizations of some of the work of Papangelou [14].

Before going on, we recall some material from the general theory of processes [2]. Let $u=(u(t, \omega))$ be a process and $A=\left(A_{t}(\omega)\right)$ an increasing process, $E A_{t}<\infty, t \in \mathbf{R}_{+}$, and $\left\{\mathcal{F}_{t}\right\}$ an increasing family of $\sigma$-fields on $\Omega$ which is right-continuous and with each $\mathcal{F}_{t}$ completed by all $P$-null sets. We write $\mathcal{l}, \mathcal{P}$ for the well-measurable (resp. predictable) $\sigma$-fields on $\mathbf{R}_{+} \times \Omega$, and note that $\mathcal{P} \subset \mathcal{C}$. The accessible $\sigma$-field falls between $\mathcal{P}$ and $\mathcal{W}$, but will be omitted from our discussion.

Writing $w(u)$ and $p(u)$ for the well-measurable and predictable projections of the process $u$, the dual well-measurable (resp. predictable) projection of the increasing process $A$ is defined as follows: $A^{w}$ (resp. $A^{p}$ ) is the unique well-measurable (resp. predictable) increasing process such that

$$
E \int_{0}^{\infty} w(u)(s, \omega) d A_{s}(\omega)=E \int_{0}^{\infty} u(s, \omega) d A_{s}^{w}(\omega), \quad u \in\left(B_{+} \otimes \mathcal{F}\right)_{+},
$$

and similarly for $A^{p}$. For an increasing process, we note that well-measurability is equivalent to being adapted.

For an integrable RAF $\alpha$, we now denote by $\alpha^{\#}$ (resp. $\alpha^{*}$ ) the dual wellmeasurable (resp. predictable) projection as defined above. 
(27) Theorem. The increasing processes $\alpha^{\#}, \alpha^{*}$ are AF's whose Palm measures are

$$
E_{a^{\sharp}}(\xi)=E_{a}\left(\xi^{\#}\right), \quad E_{\alpha^{*}}(\xi)=E_{a}\left(\xi^{*}\right), \quad \xi \in\left(\mathcal{F}^{0}\right)_{+} .
$$

Proof. It suffices to treat the predictable case, the other being entirely analogous, even somewhat easier. Suppose, for the moment, that $\alpha^{*}$ is an AF. Let $\xi \in\left(\mathcal{F}^{0}\right)_{+}$. Then $p(\xi \circ \theta)=\xi^{*} \circ \theta$, and the predictable version of (26) gives

$$
E \int_{0}^{\infty} e^{-s} \xi_{0} \theta_{s} \alpha^{*}(d s)=E \int_{0}^{\infty} e^{-s} \xi^{*} \circ \theta_{s} \alpha(d s),
$$

i.e. (28) holds. To show $a^{*}$ is an AF, it is enough to establish

$$
E\left[\alpha_{t+s}^{*}-\alpha_{t}^{*} ; A\right]=E\left[\alpha_{s}^{*} \circ \theta_{t} ; A\right], \quad A \in \mathcal{F} \text {. }
$$

The left side of (29) can be written as

$$
\begin{aligned}
& E \int_{0}^{\infty} I_{(t, t+s]}(r) I_{A}(\omega) \alpha^{*}(d r, \omega)=E \int_{0}^{\infty} p\left(I_{(t, t+s]} I_{A}\right) d \alpha \\
& =E \int_{0}^{\infty} I_{(t, t+s]}(r) P\left(A \mid \mathcal{F}_{r_{-}}\right) \alpha(d r) \\
& =E \int_{0}^{s} P\left(A \mid \mathcal{F}_{(r+t)}\right) \alpha\left(d r, \theta_{t} \omega\right) \text {, }
\end{aligned}
$$

where $P\left(A \mid \mathcal{F}_{r-}\right)$ denotes the left-continuous modification of the martingale $P\left(A \mid \mathcal{F}_{r}\right)$. Using stationarity of the flow, it is easy to prove that, for each $r, t, P\left(A \mid \mathcal{F}_{(t+r)-}\right)=P\left(\theta_{t} A \mid \mathcal{F}_{r-}\right) \circ \theta_{t}$ a.s., and so, formally, the last displayed expression becomes

$$
=E \int_{0}^{s} P\left(\theta_{t} A \mid \mathcal{F}{ }_{r}\right) \alpha(d r)=E \int_{0}^{\infty} I_{\theta_{t} A} \alpha^{*}(d r)=E\left[\alpha_{s}^{*} \circ \theta_{t} ; A\right] .
$$

The problem is to show that $P\left(\theta_{t} A \mid \mathcal{F}_{r-}\right) \circ \theta_{t}$ may be chosen indistinguishable from $P(A \mid \mathcal{F}(t+r)$ ) , as $r$ varies. However, both processes are a.s. leftcontinuous in $r$, hence are indistinguishable. Q.E.D.

Since Palm measures determine AF's (up to indistinguishability):

(30) Corollary. An AF a is adapted (resp. predictable) iff $E_{\alpha}(\xi)=$ $E_{\alpha}\left(\xi^{\#}\right)$ (resp. $E_{\alpha}(\xi)=E_{\alpha}\left(\xi^{*}\right)$ ) for every $\xi \in\left(\mathcal{F}^{0}\right)_{+}$.

Notes. (1) If $\alpha$ is adapted, then $\alpha$ will be predictable iff $E_{a}(\xi)=$ $E_{a}\left(\xi^{*}\right)$ for all $\xi \in\left(\mathcal{F}_{0+}^{0}\right)_{+}$since the Palm measure of an adapted AF is completely determined by its action on $\mathcal{F}_{0+}^{0}$.

(2) Since $P_{a}^{-}$kills polar sets in $\mathcal{F}_{0-}^{0}$, there exists (by $(10)(a)$ ) a predictable AF $\beta$ such that $P_{\beta}^{-}=P_{a}^{-}$; in fact, $\beta=\alpha^{*}$ since $E_{\beta}(\xi)=E_{\beta}\left(\xi^{*}\right)=$ $E_{a}\left(\xi^{*}\right)=E_{a^{*}}(\xi)$ for $\xi \in\left(\mathcal{F}^{0}\right)_{+}$. 
(31) Corollary. Additive projection (see $\$ 1$ ) preserves well-measurability (resp. predictability).

We consider next the splitting of an AF $\alpha$ into the sum of a continuous AF $\alpha_{c}$ and a purely discrete AF $\alpha_{d}$, i.e. the measure $a_{c}(d t, \omega)$ has no atoms for each $\omega \in \Omega$, whereas $\alpha_{d}(d t, \omega)$ is the sum of countably many point masses depending on $\omega \in \Omega$. The corresponding Palm measures are denoted $P_{c}, P_{d}$. Given $a, \alpha_{c}$ and $a_{d}$ are obtained from the usual decomposition of a measure into a continuous plus a discrete piece. If $\alpha$ is adapted, $\alpha_{c}$ and $a_{d}$ will be likewise, and $\alpha_{c}$ will be predictable; if $a$ is predictable, $a_{d}$ will be also. Let $A \in \mathcal{F}^{0}$ have an a.s. countable spoor $S_{A}$. Clearly $P_{c}(A)=0$. In particular, this is the case when $A$ is semipolar.

Now consider the discrete part $\alpha_{d}$. Denote the mass on $\{0\}$ by $\Delta: \Delta(\omega)$ $=\alpha_{d}(0, \omega)-\alpha_{d}(0-, \omega)=-\alpha_{d}(0-, \omega)$ (there is no restriction (see $\$ 0$ ) in assuming $\left.\Delta \in\left(\mathfrak{F}^{0}\right)_{+}\right)$; also let $\Delta_{t}(\omega)=\Delta \circ \theta_{t}(\omega)$ be the mass on $\{t\}$. It is well known that $P_{d}$ is supported on the set $\Omega_{d}=\{\Delta>0\}$. Writing $\Omega_{d}=$ $\bigcup_{n=1}^{\infty}\{\Delta>1 / n\}$ and recalling that $\alpha_{d}$ is finite on compacts, we see that $\Omega_{d}$ is semipolar.

When $\alpha$ is predictable, we get a finer decomposition: we can then show that $P_{d}$ is supported by a semipolar set in $\mathcal{F}_{0-}^{0}$, and this in turn will lead to the decomposition promised in $\$ 1$.

(32) Lemma. The Palm measure of a purely discrete, predictable AF a is carried by a semipolar set in $\mathcal{F}_{0-}^{0}$.

Proof. We choose for a the "perfect version" described in $\$ 0$. The process $\Delta \circ \theta_{t}=a(t)-a(t-)$ is then predictable, and is therefore indistinguishable from its predictable projection $\Delta^{*} \circ \theta_{t}$. It follows that the set $N^{*}$ $=\left\{\Delta^{*}>0\right\}$ (which is in $\mathcal{F}_{0_{-}}^{0}$ ) is semipolar since its spoor is a.s. the same as the spoor of $\Omega_{d}$, and $P_{a}\left(\Delta^{*}=0\right)=0$, since $P$ alm measures fail to distinguish indistinguishable processes. The set $N^{*}$ is thus the one required by the theorem.

The following is now immediate upon recalling our remarks in $\$ 1$ :

(33) Theorem. A finite measure $Q$ as in (9) has a decomposition $Q=$ $P_{c}^{-}+P_{d}+\mu$ where $P_{c}^{-}$charges no semipolar set in $\mathcal{F}_{0-}^{0}, P_{d}^{-}$lives on a semipolar, but charges no polar set in $\mathcal{F}_{0-}^{0}$, and $\mu$ lives on a polar set in $\mathcal{F}_{0-}^{0}$.

As a consequence of (33), we obtain the following refinements of the results in $\$ 1$ :

(34) Corollary. (a) A finite measure $Q$ on $\mathcal{F}^{0}$ is the Palm measure of 
a continuous $A F$ iff $Q$ charges no semipolar set.

(b) For an AF a, $a^{*}$ is continuous iff $P_{a}$ charges no semipolar set in $\mathcal{F}_{0-}^{0}$.

Part (a) follows by taking the trivial filtration $\mathcal{F}_{t}^{0} \equiv \mathcal{F}^{0}$ in (33), and part (b) from (33) and the note following (30).

A similar situation obtains for discrete AF's:

(35) Corollary. An AF a is purely discrete iff its Palm measure is carried by a semipolar set.

If $N$ is semipolar, it is contained in the union of thin sets $B_{n}$, so we may consider $N$ thin. By the argument in the proof of (22), the spoor $S_{N}(\omega)$ will be unbounded in both directions a.s. Since $N$ is thin, we may enumerate the points of $S_{N}(\omega): \ldots R_{-1}(\omega)<R_{0}(\omega) \leq 0<R_{1}(\omega)<\ldots$, and we have $R_{n+1}=R_{n}+R_{1} \circ \theta_{R_{n}}$ for each integer $n$. Define $\nu(d t, \omega)$ as the measure which puts unit mass on each $R_{n}(\omega)$. We leave it to the reader to check that $\nu$ is an $A F$ and that $\alpha(d t, \omega) \ll \nu(d t, \omega)$ for almost every $\omega \in \Omega$ (to show this it suffices to check $\left.Q \ll P_{\nu}\right)$. The general semipolar case is an easy extension of this method.

We conclude with a characterization of the absolute continuity of $\alpha^{*}$ in terms of $P_{a}$ similar to that given in [14] for point processes.

(36) Theorem. The following three statements are equivalent:

(a) $\alpha^{*}$ is a.s. absolutely continuous (i.e. $\left.\alpha^{*}(d t, \omega) \ll d t\right)$;

(b) $P_{a} \ll P$ on $\mathcal{F}_{0-}^{0}$;

(c) $t^{-1} E\left(\alpha(t) \mid \mathcal{F}_{0}\right)$ converges in $L^{1}$-norm as $t \downarrow 0$.

Proof. Suppose $\alpha^{*}(d t, \omega)=\xi(t, \omega) d t$. Then, for any $A \in \mathcal{F}_{0-}^{0}, P_{a}(A)=$ $P_{a^{*}}(A)=E\left[\int_{0}^{1} \xi\left(t, \theta_{-t} \omega\right) d t ; A\right]$ which proves $(\mathrm{a}) \Rightarrow(\mathrm{b})$. Conversely, assuming (b), we can write $d P_{a}^{-}=\xi d P$ for some $\xi \in\left(\mathcal{F}_{0-}^{0}\right)_{+}$. Now for any $\eta \epsilon$ $\left(\mathcal{F}^{0}\right)_{+}, E_{a^{*}}(\eta)=E_{a}\left(\eta^{*}\right)=E\left(\xi \eta^{*}\right)$ since $\eta^{*} \in\left(\mathcal{F}_{0-}^{0}\right)_{+}$. If $\eta=0$ a.s., the same is true of $\eta^{*}$, hence $P a^{*} \ll P$ and we can conclude that $\alpha^{*}(d t, \omega)=$ $\xi \circ \theta_{t}(\omega) d t$, since both are predictable and have the same Palm measure on $\mathcal{F}_{0-}^{0}$. Thus $(\mathrm{b}) \Rightarrow$ (a).

We next show that (a) is equivalent to (c). Recall the local ergodic theorem [9] which states that, if $\xi \in L^{1}\left(\Omega, \mathcal{F}^{0}, P\right), t^{-1} \int_{0}^{t} \xi \circ \theta_{s} d s \rightarrow \xi$ (as $t \downarrow 0)$ a.s. and in $L^{1}$. Now from the definition of $\alpha^{*}$ we have $E\left(\alpha(t) \mid \mathcal{F}_{0}\right)=$ $E\left(\alpha^{*}(t) \mid \mathfrak{F}_{0}\right)$ (see [2, VT 37]), and, assuming (a), we have

$$
t^{-1} E\left(\alpha(t) \mid \mathcal{F}_{0}\right)=t^{-1} E\left(\int_{0}^{t} \xi \circ \theta_{s} d s \mid \mathcal{F}_{0}\right)
$$


for some $\xi \in L^{1}$. But $t^{-1} \int_{0}^{t} \xi \circ \theta_{s} d s \rightarrow \xi\left(L^{1}\right)$, so the right member of (37) converges in $L^{1}$ to $\xi$ as well.

Conversely, assume that $t^{-1} E\left(a(t) \mid \mathfrak{F}_{0}\right)$ converges in $L^{1}$ to some $\xi$, which we may take in $\mathcal{F}_{0-}^{0}$. Again using $E\left[\alpha(t)-\alpha(s) \mid \mathcal{F}_{s}\right]=E\left[\alpha^{*}(t)-\alpha^{*}(s) \mid \mathcal{F}_{s}\right]$, $s \leq t$, we will show

$$
E \int_{0}^{1} Y_{s} a^{*}(d s)=E \int_{0}^{1} Y_{s} \xi \circ \theta_{s} d s
$$

for every continuous, adapted, bounded process $Y_{s}$. Equation (38) then extends to all predictable processes $Y$; since $\alpha^{*}(t)$ and $\int_{0}^{t} \xi \circ \theta_{s} d s$ are each predictable, (38) implies $a^{*}(t)=\int_{0}^{t} \xi \circ \theta_{s} d s$ for all $t$, a.s.

Before proving (38), we require

(39) Lemma. For $0 \leq \xi \in L^{1}$ and $Y$ as described in (38),

$$
\lim _{n \rightarrow \infty} n^{-1} \sum_{k=0}^{n-1} Y_{k / n} \xi \circ \theta_{k / n}=\int_{0}^{1} Y_{s} \xi \circ \theta_{s} d s \quad \text { (in } L^{1} \text { ). }
$$

The $L^{1}$-norm of the difference of the two members of (40) may be written

$$
\left\|n^{-1} \sum_{0}^{n-1}\left(Y_{k / n}{ }^{\xi} \circ \theta_{k / n}-n \int_{k / n}^{(k+1) / n} Y_{s} \xi \circ \theta_{s} d s\right)\right\| \leq C_{n}+D_{n} \text {, }
$$

where

$$
\begin{gathered}
C_{n}=\left\|n^{-1} \sum_{0}^{n-1} Y_{k / n}\left(\xi \circ \theta_{k / n}-n \int_{k / n}^{(k+1) / n} \xi \circ \theta_{s} d s\right)\right\|, \\
D_{n}=\left\|\sum_{0}^{n-1} \int_{k / n}^{(k+1) / n}\left(Y_{k / n}-Y_{s}\right) \xi \circ \theta_{s} d s\right\|
\end{gathered}
$$

Let $\left|Y_{s}(\omega)\right| \leq M<\infty$ for all $s, \omega$. Then

$$
C_{n} \leq \frac{M}{n} \sum_{0}^{n-1}\left\|\xi-n \int_{0}^{1 / n} \xi \circ \theta_{s} d s\right\| \rightarrow 0
$$

by the local ergodic theorem. Also,

$$
\begin{aligned}
D_{n}^{\prime} & \equiv\left|\sum_{0}^{n-1} \int_{0}^{1 / n}\left(Y_{k / n}-Y_{k / n+s}\right) \xi \circ \theta_{s} \circ \theta_{k / n} d s\right| \\
& \leq 2 M \sum_{0}^{n-1} \int_{0}^{1 / n} \xi \circ \theta_{s+k / n} d s=2 M \int_{0}^{1} \xi \circ \theta_{s} d s
\end{aligned}
$$

since $\xi \geq 0$; hence $D_{n}^{\prime}$ is dominated by an $L^{1}$ function. Now, for $\omega \in \Omega$ fixed, $Y(\omega)$ is uniformly continuous on $[0,1]$; hence, given $\epsilon>0, \mid Y_{k / n}(\omega)-$ 
$Y_{k / n+s}(\omega) \mid \leq \epsilon$ for all sufficiently large $n$, all $k \leq n-1$, and all $s$ in $[0,1 / n]$. Thus $D_{n}^{\prime} \leq \epsilon \int_{0}^{1} \xi \circ \theta_{s} d s$ for $n$ large; by dominated convergence, $D_{n} \rightarrow 0$ and (39) is proven.

Returning to the proof of (38), we have only to show

$$
E \int_{0}^{1} Y_{s} a^{*}(d s)=\lim _{n \rightarrow \infty} E n^{-1} \sum_{0}^{\dot{n}-1} Y_{k / n} \xi \circ \theta_{k / n}
$$

Let

$$
\begin{aligned}
A_{n}^{\prime} & =\left|\int_{0}^{1} Y_{s} \alpha^{*}(d s)-\sum_{0}^{n-1} Y_{k / n}\left(\alpha^{*}(k+1) / n-\alpha^{*}(k / n)\right)\right| \\
& =\left|\sum_{0}^{n-1} \int_{k / n}^{(k+1) / n}\left(Y_{s}-Y_{k / n}\right) \alpha^{*}(d s)\right| .
\end{aligned}
$$

Since $|Y| \leq M$, we find $A_{n}^{\prime} \leq 2 M a^{*}(1) \in L^{1}$; on the other hand, given $\epsilon>0$, $A_{n}^{\prime} \leq \epsilon \alpha^{*}(1)$ for all sufficiently large $n$, by a uniform continuity argument such as the one above. Hence by dominated convergence $E A_{n}^{\prime} \rightarrow 0$ and we conclude

$$
\left.\int_{0}^{1} Y_{s} \alpha^{*}(d s)=\lim _{n \rightarrow \infty} \sum_{0}^{n-1} Y_{k / n}\left(\alpha^{*}(k+1) / n-\alpha^{*}(k / n)\right) \quad \text { (in } L^{1}\right) .
$$

Now $\alpha^{*}(k+1) / n-\alpha^{*}(k / n)=\alpha_{1 / n}^{*} \circ \theta_{k / n}$. Also

$$
\begin{aligned}
& \left|E\left[\sum_{0}^{n-1} Y_{k / n} \alpha_{1 / n}^{*} \circ \theta_{k / n}-n^{-1} \sum_{0}^{n-1} Y_{k / n} \xi \circ \theta_{k / n}\right]\right| \\
& =\left|E\left[\sum_{0}^{n-1} Y_{k / n} E\left(\alpha_{1 / n}^{*} \mid \mathcal{F}_{0}\right) \circ \theta_{k / n}-n^{-1} \sum_{0}^{n-1} Y_{k / n} \xi \circ \theta_{k / n}\right]\right| \\
& =\left|\sum_{0}^{n-1} E Y_{k / n} \circ \theta_{-k / n}\left(E\left(\alpha_{1 / n}^{*} \mid \mathcal{F}_{0}\right)-\xi / n\right)\right| \\
& \leq \frac{M}{n} \sum_{0}^{n-1} E\left|n E\left(\alpha_{1 / n}^{*} \mid \mathcal{F}_{0}\right)-\xi\right| \\
& =M\left\|n E\left(\alpha_{1 / n}^{*} \mid \mathcal{F}_{0}\right)-\xi\right\| \rightarrow 0 \text { by assumption. }
\end{aligned}
$$

Putting (40), (41) and (42) together, we finally obtain (38).

\section{REFERENCES}

1. R. M. Blumenthal and R. K. Getoor, Markov processes and potential theory, Pure and Appl. Math., vol. 29, Academic Press, New York and London, 1968. MR $41 \# 9348$. 
2. C. Dellacherie, Capacités et processus stochastiques, Springer-Verlag, Berlin and New York, 1972.

3. H. Föllmer, On the representation of semimartingales, Ann. Probability 1 (1973), 580-589.

4. D. Geman and J. Horowitz, Occupation times for smooth stationary processes, Ann. Probability 1 (1973), 131-137. 232.

5. - Remarks on Palm measures, Ann. Inst. H. Poincaré 9 (1973), 215-

6. - Random shifts which preserve measure, Proc. Amer. Math. Soc. 49 (1975), 143-150.

7. A. Hanen, Processus ponctuels stationnaires et flots spéciaux, Ann. Inst. H. Poincaré Sect. B 7 (1971), 23-30. MR 46 \#2727.

8. J. de Sam Lazaro and P. A. Meyer, Méthodes des martingales et théorie des flots, Z. Wahrscheinlichkeitstheorie und Verw. Gebiete 18 (1971), 116-140.

9. - Questions de théorie des flots, Université de Strasbourg, Séminaire de Probabilités.

10. B. Maisonneuve, Ensembles régénératifs, temps locaux et subordinateurs, Lecture Notes in Math., no. 191, Springer-Verlag, Berlin and New York, 1971.

11. J. Mecke, Stationäre zufällige Masse auf lokalkompakten Abelschen Gruppen, Z. Wahrscheinlichkeitstheorie und Verw. Gebiete 9 (1967), 36-58. MR 37 \#3611.

12. - Invarianzeigens chaften allgemeiner Palmsche Masse (to appear).

13. P. A. Meyer, Probability and potentials, Blaisdell, Waltham, Mass., 1966. MR 34 \#5119.

14. F. Papangelou, Integrability of expected increments of point processes and a related random change of scale, Trans. Amer. Math. Soc. 165 (1972), 483-507. MR 47 \#2654.

15. K. R. Parthasarathy, Probability measures on metric spaces, Probability and Math. Statist., no. 3; Academic Press, New York, 1967. MR 37 \#2271.

16. D. Revuz, Mesures associées aux fonctionnelles additives de Markov. I, Trans. Amer. Math. Soc. 148 (1970), 501-531. MR 43 \#5611.

17. H. Totoki, Time changes of flows, Mem. Fac. Sci. Kyushu Univ. Ser. A 20 (1966) 27-55. MR $34 \# 1488$.

DEPARTMENT OF MATHEMATICS AND STATISTICS, UNIVERSITY OF MASSACHUSETTS, AMHERST, MASSACHUSETTS 01002 